\title{
Color Doppler analysis of female reproductive vasculature in Behçet's disease
}

\author{
M.E. Tezcan', O. Temizkan², A. Ozderya ${ }^{3}$, M. Melikoglu4 , K. Aydin'3, \\ M. Sargin ${ }^{5}$, S. Temizkan ${ }^{3}$ \\ ${ }^{1}$ Department of Rheumatology, Kartal Dr. Lutfi Kirdar Training and Research Hospital, Istanbul, Turkey; \\ 2Department of Gynecology and Obstetrics, Sisli Etfal Training and Research Hospital, Istanbul, Turkey; \\ ${ }^{3}$ Department of Endocrinology and Metabolism, Kartal Dr. Lutfi Kirdar Training and Research Hospital, \\ Istanbul, Turkey; ${ }^{4}$ Department of Family Medicine and Diabetes, Kartal Dr. Lutfi Kirdar Training \\ and Research Hospital, Istanbul, Turkey; ${ }^{5}$ Department of Family Medicine and Diabetes, \\ Kartal Dr. Lutfi Kirdar Training and Research Hospital, Istanbul, Turkey
}

\section{SUMMARY}

Behçet's disease (BD) may affect female reproductive vasculature. We aimed to evaluate Doppler sonographic characteristics of female reproductive vasculature and also ovarian volume, endometrial thickness (EMT) and antral follicle count of BD patients in comparison with a healthy control group.

Seventeen premenopausal women aged between 18-45 years with BD, and a control group of 31 age- and body mass index-matched healthy women was included in the study. Uterine, spiral and intraovarian artery blood flow were examined by Doppler sonography in the late follicular phase. Resistance index, pulsatility index and systolic/diastolic ratio were recorded together with ovarian volume, EMT and antral follicle count. In particular this is a pilot study including the evaluation of the spiral and uterine arteries in BD.

Doppler sonographic parameters, ovarian volume, EMT and antral follicle count of BD patients and healthy controls were not found to be statistically different.

As a result of our analysis, we found similar Doppler sonographic features of both BD patients and the control group. Further studies conducted on a larger sample population with more aggressive BD symptoms may reveal the actual effect of BD on the female reproductive system.

Key words: Behçet's disease; Doppler sonography; intraovarian artery; spiral artery; uterine artery.

\section{INTRODUCTION}

ehçet's disease (BD) is an inflammatory disorder of unknown cause, characterized by recurrent oral and/or genital aphthous ulcers accompanied by cutaneous, ocular, articular, gastrointestinal, and/ or central nervous system inflammatory lesions (1). Apart from genital aphthous ulcers, BD can cause ovarian vein thrombosis (2), placental necrotizing vasculitis, decidual villitis (3), and diminished ovarian reserve (4) in the female reproductive system. BD may have an impact on the female reproductive vasculature since BD vasculitis can also affect arteries or veins (5). In the female reproductive system, the availability of an adequate vascular supply provides endocrine and paracrine signals which may play a key role in the regulation of follicle growth (6), while placental vasculature is also related to preeclampsia and intrauterine growth retardation (7). Therefore, BD can lead to infertility or unfavorable pregnancy due to vascular or structural changes in the female reproductive system. On the basis of the limited bulk of data available so far, BD was not found to trigger these outcomes $(8,9)$. Nevertheless, there is not sufficient and detailed research evidence on how BD affects the female reproductive vasculature. In this study, we aimed to evaluate simultaneously uterine, spiral and intraovarian artery Doppler velocimetry characteristics in comparison with the control group. Also, we examined ovar-
Corresponding author: Mehmet Engin Tezcan Department of Rheumatology, Kartal Dr. Lutfi Kirdar Trainig and Research Hospital, Kartal, Istanbul, Turkey E-mail: engintez@yahoo.com 
ian volume, endometrial thickness (EMT) and antral follicle count (AFC) of BD patients versus controls.

\section{MATERIALS AND METHODS}

\section{Participants}

In this study, we prospectively enrolled 17 premenopausal women aged between 18-45 years with BD from the Outpatient Rheumatology Clinic between June 2013 and June 2014. All the participants with BD fulfilled the 1990 Criteria for the diagnosis of the disease (10).

The exclusion criteria were menopause, current pregnancy, hypothalamo-pituitarygonadal axis dysfunction, use of a hormonal contraceptive in the previous six months, gynecological surgery, gynecological cancer, presence of an additional auto-immune disease, pelvic inflammatory disease, cardiovascular disease, diabetes mellitus, polycystic ovary syndrome, use of medications affecting the vasculature, such as antihypertensive and cardiovascular drugs.

This patient population was compared with a control group of 31 age- and body mass index (BMI)-matched healthy women. The study was approved by the local Ethics Committee and all women participating in the study gave their written informed consent.

The study was performed in accordance with the Declaration of Helsinki.

All the participants underwent a detailed evaluation after enrollment. The medical history of the patients related to BD including organ involvement, past and current treatments, disease duration were recorded from from an electronic database. In addition, anthropometric measurements of participants including height, weight were recorded and their BMIs were calculated as kilograms per square meter. For each participant, blood pressure was measured after a 15-min rest with an automated digital sphygmomanometer (Omron Healthcare, Kyoto, Japan) in supine position and the average of the measurements from both arms was included in the analyses.

As a part of the evaluations, blood samples of the subjects were taken between 08:00 and 09:00 am after an overnight 12-h fasting period during the early follicular phase (Day 2-5).

These blood samples were later tested for glucose, insulin, follicle stimulating hormone (FSH), luteinizing hormone (LH), estradiol, testosterone, sex hormone binding globulin (SHBG), dehydroepiandrosteronesulphate (DHEAS).

Table I - Clinical and biochemical features of patients with Behçet's disease and healthy controls.

\begin{tabular}{|l|l|l|l|}
\hline & $\begin{array}{l}\text { Behçet's disease } \\
\text { patients }(\mathbf{n}=\mathbf{1 7})\end{array}$ & $\begin{array}{l}\text { Controls } \\
(\mathbf{n}=\mathbf{3 1})\end{array}$ & $\mathbf{p}$ \\
\hline Age (years) & $35.6 \pm 6.6$ & $34.0 \pm 5.7$ & 0.39 \\
\hline BMI $\left(\mathrm{kg} / \mathrm{m}^{2}\right)$ & $27.62 \pm 4.9$ & $27.60 \pm 2.97$ & 0.78 \\
\hline Smoking $\mathrm{n}(\%)$ & $6(35.2)$ & $5(23.1)$ & 0.67 \\
\hline Systolic BP $(\mathrm{mmHg})$ & $113.00 \pm 14.88$ & $118.58 \pm 11.88$ & 0.36 \\
\hline Diastolic BP $(\mathrm{mmHg})$ & $76.15 \pm 7.08$ & $78.83 \pm 8.10$ & 0.35 \\
\hline TSH $(\mu \mathrm{U} / \mathrm{mL})$ & $1.22 \pm 0.53$ & $1.62 \pm 0.88$ & 0.19 \\
\hline FSH $(\mathrm{IU} / \mathrm{L})$ & $6.89 \pm 3.29$ & $6.94 \pm 3.19$ & 0.97 \\
\hline LH $(\mathrm{IU} / \mathrm{L})$ & $5.32 \pm 2.99$ & $5.54 \pm 1.97$ & 0.48 \\
\hline Estradiol $(\mathrm{pg} / \mathrm{mL})$ & $76.60 \pm 67.8$ & $55.60 \pm 26.91$ & 0.88 \\
\hline Testosterone $(\mathrm{ng} / \mathrm{dL})$ & $23.61 \pm 9.85$ & $27.97 \pm 12.75$ & 0.67 \\
\hline DHEAS $(\mu \mathrm{g} / \mathrm{dL})$ & $152.14 \pm 100.94$ & $170.95 \pm 113.78$ & 0.23 \\
\hline SHBG $(\mathrm{nmol} / \mathrm{dL})$ & $56.67 \pm 28.89$ & $58.08 \pm 24.30$ & 0.73 \\
\hline
\end{tabular}

BMI, body mass index; BP, blood pressure; TSH, thyroid stimulating hormone; FSH, follicle stimulating hormone; LH, luteinizing hormone; DHEAS, dehydroepiandrosteronesulphate; SHBG, sex hormone binding globu 
Table II - Doppler sonographic findings of Behçet's disease patients and controls.

\begin{tabular}{|l|l|l|l|}
\hline & $\begin{array}{l}\text { Behçet's disease } \\
\text { patients } \mathbf{( n = 1 7 )}\end{array}$ & $\begin{array}{l}\text { Control } \\
\mathbf{( n = 3 1 )}\end{array}$ & $\mathbf{P}$ \\
\hline Endometrial thickness $(\mathrm{mm})$ & $7.54 \pm 1.54$ & $7.12 \pm 1.81$ & 0.46 \\
\hline Spiral artery PI & $1.08 \pm 0.33$ & $1.25 \pm 0.47$ & 0.26 \\
\hline Spiral artery RI & $0.58 \pm 0.13$ & $0.63 \pm 0.12$ & 0.29 \\
\hline Spiral artery S/D & $2.72 \pm 0.92$ & $3.05 \pm 1.07$ & 0.31 \\
\hline Intraovarian artery PI & $0.83 \pm 0.24$ & $0.93 \pm 0.30$ & 0.25 \\
\hline Intraovarian RI & $0.51 \pm 0.15$ & $0.54 \pm 0.10$ & 0.64 \\
\hline Intraovarian S/D & $2.07 \pm 0.46$ & $2.31 \pm 0.62$ & 0.71 \\
\hline Uterine artery PI & $2.41 \pm 0.71$ & $2.38 \pm 0.62$ & 0.90 \\
\hline Uterine artery RI & $0.83 \pm 0.05$ & $0.82 \pm 0.09$ & 0.64 \\
\hline Uterine artery S/D & $6.89 \pm 2.75$ & $6.49 \pm 3.09$ & 0.69 \\
\hline Antral follicle count & $12.92 \pm 3.95$ & $12.90 \pm 3.51$ & 0.98 \\
\hline Low antral follicle count $(\mathrm{n})$ & 4 & 4 & 0.22 \\
\hline Mean ovarian volume $\left(\mathrm{mm}^{3}\right)$ & $7.67 \pm 2.44$ & $6.87 \pm 2.78$ & 0.44 \\
\hline
\end{tabular}

PI, pulsatility index; RI, resistive index; S/D, systole/diastole ratio.

\section{Doppler ultrasonographic evaluation}

Firstly, our pilot study evaluated the spiral and uterine arteries in BD.

The evaluation of the patients and the control group was performed by standard Bmod transvaginal ultrasonography and color Doppler ultrasonography in the morning (8:00-10:00 a.m.) during the late follicular phase (Day 5-8, after the menstrual bleeding). Uterine, spiral (subendometrial), and intra-ovarian arteries were visualized using the color Doppler technique. All ultrasonographic evaluations were conducted by one individual experienced physician (O.T.) who had no prior information about the patients, using a Siemens Acuson Antares scanner with $7 \mathrm{MHz}$ conventional grayscale endovaginal probe in the lithotomy position with empty bladder.

Doppler ultrasounds, both in the color and the spectral modes, had a frequency of $5 \mathrm{MHz}$. The high-pass filter used in the uterine artery was $125 \mathrm{~Hz}$, whereas for the intraovarian and spiral artery it was $50 \mathrm{~Hz}$. The setting was adjusted to ensure the highest sensitivity for the low velocity flow, pulse repetition frequency was 0.5$1.2 \mathrm{kHz}$, color Doppler gain was reduced until all color artifacts disappeared. Pulsed Doppler signals were obtained using the 1-2 mm volume cursor. Uterine, spiral and intra-ovarian arteries resistance indexes (RI), pulsatility indexes (PI) and systolic/ diastolic ratio (S/D) were measured at this stage. The details of the data analysis with intra-observer reliability were as previously described (11).

For all participants, ovarian volume and morphology were evaluated in both ovaries. Endometrial thickness (mm) was measured at the thickest point along the sagittal plane (double layer), while ovarian volume $\left(\mathrm{cm}^{3}\right)$ was estimated by using a formula (length $\mathrm{x}$ width $\mathrm{x}$ height $\mathrm{x} 0.523$ ). The mean volume was calculated and considered for further analysis. Lastly, ovarian morphology was assessed by counting the number of antral follicles with a diameter between $2-9 \mathrm{~mm}$ considered to be in the AFC. We also calculated the sum of antral follicles in both ovaries (total number of follicles in both ovaries).

\section{Assays}

Fasting plasma glucose (FPG) was measured with the glucose oxidase method (Olympus AU 2700, Olympus America Inc., Melville, NY, USA).

Insulin levels were measured with the immunoradiometric assay method (Diasource, Louvain, Belgium). FSH, LH, estradiol, testosterone, SHBG and DHEAS 
were measured by UniCelDxl 600 Acces Immunoassay System (Beckmann Coulter, Florida, USA).

\section{Statistical analyses}

Statistical analyses were carried out using SPSS Version 17.0 (SPSS Inc., Chicago, IL, USA). In order to determine whether the data is normally distributed, the Kolmogorov-Smirnov test was performed. In addition, the comparison of continuous variables between patients and controls was done by using Student $t$ test and/or MannWhitney $\mathrm{u}$ test depending on the normality of the distribution. The comparison of the categorical variables was performed by using the Chi-square test. Correlation analyses were performed by using Spearman/ Pearson correlation tests. The results were expressed as mean \pm standard deviation or median (min-max) when appropriate and $\mathrm{p}<0.05$ was considered to be statistically significant.

\section{RESULTS}

\section{Subjects}

The clinical and biochemical features of the participants are reported in Table I. According to our results, no difference emerged between BD patients and controls regarding demographic, anthropometric features, and hormonal analysis. None of the BD patients was infertile. Also, menstrual cycles were irregular in $2 \mathrm{BD}$ patients (in one the cycle was $<26$ days, whereas in the other it was $>35$ days). On the contrary, all controls had regular menstrual cycles and 9 of them were nulliparous.

\section{Doppler sonographic findings and hormones}

Doppler sonographic findings of the participants are reported in Table II. Doppler sonographic parameters, ovarian volume, EMT and antral follicle count of BD patients and controls were not found to be statistically different.

Behçet's disease patients' disease features Median disease duration at the time of recruitment was 30 (7-324) months. Evalu- ations show that all patients had oral aphthous ulcers. Genital ulcers were present in $64.7 \%(11 / 17)$, erythema nodosum in $58.8 \%(10 / 17)$, pathergy positivity in $29.5 \%$ (5/17). Also, 2 patients reported a history of arthritis, while 3 patients had a history of organ involvement. One patient had posterior uveitis, 1 patient had neurological parenchymal involvement and 1 patient had posterior uveitis, pulmonary artery aneurism and neurological parenchymal involvement. These three patients with systemic involvement were in remission. Their medical therapies consisted of colchicine $(92.8 \%)$, azathioprine $(14.2 \%)$, steroids (14.2\%), hydroxychloroquine (7.1\%), sulphasalazine $(7.1 \%)$ and methotrexate $(7.1 \%)$ at the time of recruitment. Two patients had also a history of cyclophosphamide treatment.

\section{DISCUSSION}

$\mathrm{BD}$ is a multisystemic vasculitis and is generally known to affect the female reproductive system with genital aphthous ulcers and their associated sequelae (1). In this study, we investigated the female reproductive vasculature, which may be related to ovarian reserve, pregnancy outcome and fertility in BS patients. To our knowledge, no other study in the literature investigated uterine, spiral and intra-ovarian arteries all together. Also, this is the first pilot study that evaluated the spiral and uterine arteries in BD. We found that women with BD had similar Doppler sonographic characteristics of uterine, spiral and intra-ovarian arteries compared to control women. Additionally, ovarian volumes, AFC, EMT and serum levels of reproductive hormones were not different between BS patients and controls. The endometrial blood flow as shown by the spiral artery may have an important role in endometrial receptivity. Accordingly, in a study, both pulsatility and resistance indexes of the spiral artery were found to be significantly higher in the infertile study group than in the fertile control group (12). Also, the spiral artery S/D ratio was lower in the patients who had a pregnancy compared to those who did not 
(13). In our study, no significant difference was found in the Doppler sonographic parameters of the spiral artery between $\mathrm{BD}$ patients and controls.

In the literature the intra-ovarian blood flow in the early follicular phase of spontaneous cycles is reported to be related to the ovarian follicular response (14). Lower PI values revealing an increased blood flow were detected in the ovarian artery implying the presence of an improved supply of oxygen, nutrients, hormones and growth factors (15). Likewise, in another study, infertile patients had higher PIs in the ovarian arteries compared to controls (16). Also both ovarian artery pulsality and resistance indices were found significantly lower in good responders in in-vitro fertilization treatments (17). Only one study published in the literature analyzes ovarian artery Doppler sonography in BD. In that study, Cil et al. found that the ovarian artery Doppler sonographic characteristics did not show any significant difference compared with controls (18). Also, hormonal parameters and ovarian volumes were similar between $\mathrm{BD}$ patients and controls. In our study, ovarian artery velocitometry parameters were not significantly different between BD patients and controls.

Uterine artery velocimetry indexes, such as RI, PI and S/D, are usually important in pregnancy $(19,20)$, but they were considered also valuable in the assessment of endometrial receptivity (21). Our results revealed that uterine artery Doppler parameters were similar to controls.

EMT is one of the predictors for fertilization. A thin endometrium defined as EMT $\leq 7 \mathrm{~mm}$ (22) is known to adversely affect reproductive performance (23). In in vitro fertilization, a trend towards lower ongoing pregnancy and living birth rates for women with EMT $\leq 7 \mathrm{~mm}$ was observed and the probability of clinical pregnancy for an EMT $\leq 7 \mathrm{~mm}$ was significantly lower compared with cases with EMT $>7 \mathrm{~mm}$ (24). In this study, $37.5 \%(6 / 16)$ of our BS patients and $50 \%(16 / 31)$ of controls had EMT $<7$ $\mathrm{mm}$, but no statistically significant difference was found between the patients with EMT $<7 \mathrm{~mm}$.
AFC was related to the ovarian reserve and aging (25). AFC lower or equal to 10 is accepted as low and associated with a diminished ovarian reserve (26). In our study, $25.0 \%$ of BD patients (4/16) and $12.0 \%$ of controls (4/31) had low AFC, but this difference proved to be significant. With a similar approach, Mont'Alverne et al. have recently analyzed the ovarian reserve in 10 BD patients, determining anti-mullerian hormone $(\mathrm{AMH})$ levels, ovarian volumes, AFC and hormonal parameters (4). They found no difference for ovarian volume, $\mathrm{AFC}$ and hormonal parameters like in our study except for AMH levels, which were significantly lower in BD patients. They suggested that BD patients may have a diminished ovarian reserve.

As an aside, the ovarian volume was also accepted as one of the markers of ovarian reserve and is known to decrease with aging (27). We did not detect any difference between ovarian volumes of BD patients and controls like Cil et al. (18) and Mont'Alverne et al. (4).

There are several limitations in our study. First of all, unfortunately, our study included a relatively small number of patients, but it is a pilot study evaluating the spiral and uterine arteries in BD. Secondly, BD patients had a mild clinical picture; only 3 patients had systemic involvement other than muco-cutaneous symptoms and 1 patient has vascular involvement. In a group of patients with more aggressive systemic and vascular involvement, vascular changes in female reproductive system might be more apparent.

\section{CONCLUSIONS}

In conclusion, patients with $\mathrm{BD}$ seem to have similar Doppler sonographic features of the female reproductive system compared with controls. Further studies of a larger number of patients and focusing on patients with more aggressive BD clinical symptoms may be beneficial to reveal the actual effect of BD on the female reproductive system.

Acknowledgements: None. 
Conflict of interest: The authors reports no conflict of interest.

\section{REFERENCES}

1. Sakane T, Takeno M, Suzuki N, Inaba G. Behcet's disease. N Engl J Med. 1999; 341: 1284-91.

2. Tan JW, Howe HS, Chng HH. Ovarian vein thrombosis in Behcet disease. J Clin Rheumatol. 2012 ; 18: 89-91.

3. Hwang I, Lee CK, Yoo B, Lee I. Necrotizing villitis and decidual vasculitis in the placentas of mothers with Behcet disease. Hum Pathol. 2009; 40: 135-8.

4. Mont'Alverne AR, Yamakami LY, Goncalves CR, Baracat EC, Bonfa E, Silva CA. Diminished ovarian reserve in Behcet's disease patients. Clin Rheumatol. 2014 [Epub ahead of print].

5. Jennette JC, Falk RJ, Bacon PA, Basu N, Cid MC, Ferrario F, et al. 2012 revised International Chapel Hill Consensus Conference Nomenclature of Vasculitides. Arthritis Rheum. 2013; 65: 1-11.

6. Redmer DA, Reynolds LP. Angiogenesis in the ovary. Rev Reprod. 1996; 1: 182-92.

7. Kalache KD, Duckelmann AM. Doppler in obstetrics: beyond the umbilical artery. Clin Obstet Gynecol. 2012; 55: 288-95.

8. Uzunaslan D, Saygin C, Hatemi G, Tascilar K, Yazici H. No appreciable decrease in fertility in Behcet's syndrome. Rheumatology (Oxford). 2014; 53: 828-33.

9. Noel N, Wechsler B, Nizard J, CostedoatChalumeau N, Boutin du LT, Dommergues M, et al. Behcet's disease and pregnancy. Arthritis Rheum. 2013; 65: 2450-6.

10. [No authors listed]. Criteria for diagnosis of Behçet's disease. International Study Group for Behçet's disease. Lancet. 1990; 335: 1078-80.

11. Temizkan O, Temizkan S, Asicioglu O, Aydin K, Kucur S. Color Doppler analysis of uterine, spiral, and intraovarian artery blood flow before and after treatment with cabergoline in hyperprolactinemic patients. Gynecol Endocrinol. 2014:1-4.

12. Uysal S, Ozbay EP, Ekinci T, Aksut H, Karasu $\mathrm{S}$, Isik AZ, et al. Endometrial spiral artery Doppler parameters in unexplained infertility patients: is endometrial perfusion an important factor in the etiopathogenesis? J Turk Ger Gynecol Assoc. 2012; 13: 169-71.

13. Gong X, Li Q, Zhang Q, Zhu G. Predicting endometrium receptivity with parameters of spiral artery blood flow. J Huazhong Univ Sci Technolog Med Sci. 2005; 25: 335-8.

14. Zaidi J, Barber J, Kyei-Mensah A, Bekir J, Campbell S, Tan SL. Relationship of ovarian stromal blood flow at the baseline ultrasound scan to subsequent follicular response in an in vitro fertilization program. Obstet Gynecol. 1996; 88: 779-84.

15. Kim SH, Ku SY, Jee BC, Suh CS, Moon SY, Lee JY. Clinical significance of transvaginal color Doppler ultrasonography of the ovarian artery as a predictor of ovarian response in controlled ovarian hyperstimulation for in vitro fertilization and embryo transfer. J Assist Reprod Genet. 2002; 19: 103-12.

16. Tinkanen H, Kujansuu E, Laippala P. Vascular resistance in uterine and ovarian arteries: its association with infertility and the prognosis of infertility. Eur J Obstet Gynecol Reprod Biol. 1994; 57: 111-5.

17. Altundag M, Levi R, Adakan S, Goker EN, Killi R, Ozcakir HT, et al. Intraovarian stromal artery Doppler indices in predicting ovarian response. J Reprod Med. 2002; 47: 886-90.

18. Cil AP, Karabulut AA, Kocak M. Assessment of ovarian stromal artery Doppler characteristics and serum hormone levels in patients with Behcet disease. Diagn Interv Radiol. 2010; 16: 288-92.

19. Afrakhteh M, Moeini A, Taheri MS, Haghighatkhah HR, Fakhri M, Masoom N. Uterine Doppler velocimetry of the uterine arteries in the second and third trimesters for the prediction of gestational outcome. Rev Bras Ginecol Obstet. 2014; 36: 35-9.

20. Jodicke C, Maulik D, Singh P, Heitmann E, Maulik D. Role of ultrasound in pre-eclampsia. Minerva Ginecol. 2012; 64: 293-308.

21. Stener-Victorin E, Waldenstrom U, Andersson SA, Wikland M. Reduction of blood flow impedance in the uterine arteries of infertile women with electro-acupuncture. Hum Reprod. 1996; 11: 1314-7.

22. Gleicher N, Vidali A, Barad DH. Successful treatment of unresponsive thin endometrium. Fertil Steril. 2011; 95: 2123: e13-7.

23. Lebovitz O, Orvieto R. Treating patients with "thin" endometrium - an ongoing challenge. Gynecol Endocrinol. 2014; 30: 409-14.

24. Kasius A, Smit JG, Torrance HL, Eijkemans MJ, Mol BW, Opmeer BC, et al. Endometrial thickness and pregnancy rates after IVF: a systematic review and meta-analysis. Hum Reprod Update 2014; 20: 530-41.

25. Younis JS. Ovarian aging and implications for fertility female health. Minerva Endocrinol. 2012; 37: 41-57.

26. Hendriks DJ, Kwee J, Mol BW, te Velde ER, Broekmans FJ. Ultrasonography as a tool for the prediction of outcome in IVF patients: a comparative meta-analysis of ovarian volume and antral follicle count. Fertil Steril. 2007; 87: 764-75.

27. Erdem A, Erdem M, Biberoglu K, Hayit O, Arslan M, Gursoy R. Age-related changes in ovarian volume, antral follicle counts and basal FSH in women with normal reproductive health. J Reprod Med. 2002; 47: 835-9. 